CAHIERS DE

NARRATOLOGIE

\section{Cahiers de Narratologie}

Analyse et théorie narratives

15 | 2008

Récits et genres historiques

\title{
Roman historique et Histoire dans le roman
}

Quelques modalités d'intégration de l'histoire récente dans le roman espagnol de la fin du millénaire

\section{Christine Di Benedetto}

\section{(2) OpenEdition}

Journals

Édition électronique

URL : http://journals.openedition.org/narratologie/767

DOI : 10.4000/narratologie.767

ISSN : 1765-307X

Éditeur

LIRCES

Référence électronique

Christine Di Benedetto, «Roman historique et Histoire dans le roman », Cahiers de Narratologie [En ligne], 15 | 2008, mis en ligne le 14 décembre 2008, consulté le 19 avril 2019. URL : http:// journals.openedition.org/narratologie/767; DOI : 10.4000/narratologie.767

Ce document a été généré automatiquement le 19 avril 2019

\section{(c) $($ i) $(9)$}

Cahiers de Narratologie - Analyse et théorie narratives est mis à disposition selon les termes de la licence Creative Commons Attribution - Pas d'Utilisation Commerciale - Pas de Modification 4.0 International. 


\title{
Roman historique et Histoire dans le roman
}

\author{
Quelques modalités d'intégration de l'histoire récente dans le roman \\ espagnol de la fin du millénaire
}

\section{Christine Di Benedetto}

1 Dans le cadre de ce séminaire sur le récit historique, nous allons nous intéresser à un genre quasi hégémonique dans le champ contemporain des pratiques sociales du livre en Espagne, le roman. Nous pourrons envisager, pour cette raison, que ce qui s'y produit a un certain degré d'impact sur la société. Nous nous proposons de réfléchir aux diverses problématiques liées au roman historique dans ce pays, sur les trente dernières années, ainsi que le précise notre sujet : «Roman historique et Histoire dans le roman. Quelques modalités d'intégration de l'histoire récente dans le roman espagnol de la fin du millénaire ». Dans un premier temps, nous tenterons de définir le terme de "roman historique " et aborderons les questions, plus vastes, d'Histoire dans le roman et de roman de la mémoire. Il conviendra, à cet égard, de préciser les notions de passé et de présent, au regard de la situation particulière de l'Espagne autour de 1975. Nous verrons ce que cela signifie au niveau de la répartition quantitative dans le panorama romanesque espagnol. Puis nous mettrons en perspective ces deux temporalités, faisant appel pour cela à des analyses qui relèvent de la philosophie et de la sociologie, afin de cerner le lien qualitatif entre passé et présent, que le roman peut être amené à mettre en évidence. Dans un deuxième temps, nous envisagerons, à travers des exemples dans l'Espagne contemporaine, trois niveaux d'intégration de l'Histoire dans le roman. Nous ferons appel pour cela à des éléments d'analyse fictionnelle et narrative.

Roman historique?

2 Précisons qu'aujourd'hui plusieurs travaux ${ }^{1}$ montrent que la concurrence entre romancier et historien est dépassée, ce qui pousse à revoir la problématique générique. En parallèle, on assiste à un brouillage éditorial significatif en matière de classification par genre. Isabelle Touton ${ }^{2}$, dans une thèse sur le roman historique contemporain dont l'action se passe au Siècle d'Or, a montré que le flou généré par les maisons d'éditions correspond à un effacement des frontières, lequel, de manière insidieuse, s'est imposé aux 
lecteurs. Une des preuves est qu'il a déjà été assumé par une institution comme l'école, le roman historique servant de relais accepté à un enseignement historique lacunaire ou trop orienté. Umberto Eco affirme d'ailleurs que dans le roman historique «les agissements des personnages servent à mieux faire comprendre l'histoire, ce qui s'est passé, et bien qu'ils soient inventés, ils en disent plus, et avec une clarté sans pareille, sur (...) l'époque, que les livres d'histoire consacrés. ${ }^{3}$ "

3 Si nous acceptons de laisser de côté une dichotomie de principe lorsque nous nous interrogeons sur la question historiographie/roman historique, il apparaît que dans les deux cas face la question de la visibilité se pose. Que donne-t-on à voir et comment ? Cette préoccupation, il faut le noter, se conçoit dans le cadre d'un idéal d'authenticité. A ce titre, quel que soit le niveau de réussite dans le rendu de l'authentique, nous entrons sur les territoires de l'éthique ${ }^{4}$. Et ceux-ci dépendent d'une certaine image que l'on a de la réalité et de la manière dont la société se dit et se met en scène. Ce qui conduit à se demander si le contraire de la vérité est la fausseté (ou le faux) ou bien le mensonge...

Déterminer à partir de quand on peut parler de « roman historique » n'est pas chose aisée car cette terminologie recouvre plusieurs réalités.

5 On pense d'abord à une question de dosage, devant des romans dont la diégèse se déroule dans le passé et d'autres qui se passent dans le présent, mais avec le retour, plus ou moins important, d'un des protagonistes sur son passé ou sur un passé raconté. La seconde implication n'est pas tant la «quantité » de passé qu'on y trouve mais le rapport au présent. Or Isabelle Touton a montré que le roman historique dont la diégèse se déroule au Siècle d'Or, bien que lointain, a d'une certaine manière une implication sur le présent car il dénote un positionnement idéologique de la société dans laquelle il est créé et lu.

6 Nous nous intéresserons ici à la seconde catégorie de romans, ceux ancrés à la fois dans le présent et le passé, ces romans de la mémoire, que l'on pourra qualifier également d'historiques, dans lesquels on ressent un rapport plus direct et fort au présent.

7 Pour tenter de comprendre les enjeux qui sont associés au fait que l'on trouve des éléments historiques dans un roman, sans être entravés par des questions quantitatives, nous adopterons l'expression : « Histoire dans le roman ». Mais nous devrons au préalable définir ce que nous entendons par Histoire ou éléments historiques. Ce n'est que dans un second temps que nous nous pencherons sur les questions de dosage, lorsque nous étudierons diverses modalités de l'intégration de l'Histoire dans le roman espagnol de la fin du millénaire.

Histoire dans le roman

Nous utilisons le terme « Histoire » dans un sens large, en tant qu'événements précis ou situations générales, concernant des personnalités reconnues comme des acteurs anonymes, et relevant d'un passé plus ou moins récent, mais que l'on peut relier à un espace et à un temps identifiables.

9 C'est l'utilisation qui est faite de l'Histoire récente dans le roman qui nous intéresse ici. Todorov ${ }^{5}$ affirme en effet qu'il existe une différence entre la récupération du passé et son utilisation. Nous ne rechercherons donc pas la véracité historique, ni ne traquerons les indices qui révèlent cette Histoire. Nous voyons ici plutôt le roman comme un «lieu de mémoire ", comme un moment dans l'histoire des représentations d'un monde. Pour ce, il convient de refuser en premier lieu la confrontation entre mémoire et oubli qui renferme le présupposé qu'il existe une mémoire objective, comme s'il s'agissait d'un lieu où se conservent intactes les représentations du passé. En suivant les réflexions du philosophe 
espagnol Manuel $\mathrm{Cruz}^{6}$, nous considérons que la mémoire est un ensemble de pratiques visant à la construction de l'identité de chacun, de chaque société ou micro-société. Dans ce sens, elle ne conserve pas un stock d'informations, mais elle met en évidence, signale ou attire l'attention sur certaines d'entre elles.

Ce sont ces choix qui, liés à la notion d'identité sont, à notre sens, particulièrement fondamentaux pour le corpus des romans d'après 1975. Dans le présent en mutation de la Transition démocratique, présent peut-être rendu illisible par l'absence de repères, on constate la tendance à partir à la recherche d'un passé, réécrit et donc reconstruit, lisible. L'écriture de la mémoire a donc une double visée: celle d'une essence à comprendre ainsi que celle d'une identité à construire. Il semble en effet que l'on puisse définir un lien étroit entre identité et mémoire. Car la personne comme la société s'inscrivent dans la conscience d'une identité en évolution - diachronique -, en même temps qu'elles se révèlent à travers la notion d'appartenance. C'est pourquoi le processus de constitution de l'identité est multiple. Il passe par le regard de l'autre, et par diverses pratiques de reconnaissance, qui tiennent compte du relativisme historique et de l'existence de diverses formes d'identité. Ce processus se met en place par la présence d'un «ailleurs » ou d'un "autrement», définis par une réflexion ou simplement parce que des gens seront « visiblement différents ».

11 Santos Alonso signale, dans le roman de la Transition, « un engagement rénové à l'égard du réalisme et de l'homme ». En fait la réalité présente est écrite le plus souvent à travers une perception et une transcription subjectives de ce qu'elle est et provoque. Et c'est là que l'on retrouve la préoccupation d'ordre éthique, aujourd'hui sous la forme d'une sensibilité plus individualiste et parfois ironique.

Dans ce processus de quête identitaire, Manuel Cruz estime que « comprendre le présent c'est affronter le passé et s'engager vers un avenir ». A l'échelle d'une société, il n'y aurait pas d'intelligibilité du passé sans une claire perception de son propre projet d'avenir, passé et futur étant deux faces inséparables de la même pièce.

Or la situation espagnole dans les années de la démocratisation est clairement orientée vers la recherche et l'établissement consensuels de bases pour l'avenir. Rappelons que la Constitution qui régule aujourd'hui encore l'Etat espagnol date de 1978. Le cheminement oscille entre la recherche d'un universalisme longtemps refusé par volonté d'autarcie, ou retardé par une ouverture timide et non avouée, et un désir de s'implanter dans une tradition nationale renouvelée. C'est-à-dire, non plus chercher à l'extérieur du pays le sens de sa nouvelle réalité, mais se réapproprier racines et traditions, pour mieux les ouvrir au monde ensuite. C'est à ce titre que l'écriture de la mémoire peut s'avérer fondamentale.

14 Très vite on a constaté que la démocratie ne produirait pas d'elle-même des chefsd'œuvre, et l'espoir de trouver un de ceux-ci caché dans un tiroir dans l'attente de la liberté d'expression a disparu. Il s'en est suivi une crise de conscience des intellectuels du temps du desencanto ( désenchantement») et du pasotismo ( «je-m'en-foutisme»), en même temps que l'éveil d'une culture souterraine et hybride, appelée Movida. Les deux, chacun à leur manière, ont conduit à un débat philosophique sur le rôle critique de l'écrivain ou l'autonomie de la vie culturelle. C'est pourquoi nous avons aussi voulu apporter à la question de l'Histoire dans le roman quelques pistes de réflexion philosophique. 
Dans le processus de normalisation politique et intellectuel, il a très vite fallu que le lecteur (-citoyen) mais avant lui l'écrivain (-citoyen) établissent de nouveaux signes d'identité culturelle. En premier lieu, celle-ci est devenue individuelle, après l'époque de l'éthique de la clandestinité, des pratiques communautaires de tertulias ou de la référence à des "générations » (de 27, du milieu du siècle...), celle d'une certaine solidarité du monde littéraire face à la société hostile. Ensuite, l'écrivain a dû redéfinir une légitimité pour son acte créateur et forger sa place au sein de son espace de référence. Dans ce contexte, le retrait du politique a modifié les modes d'intervention de l'écrivain. Les mécanismes commerciaux nouveaux du monde éditorial ont obligé à des négociations pour que puisse être conservée l'indépendance de la création. Ils ont aussi fait naître des débats où interviennent éditeurs, critiques et écrivains et ont abouti à un entrelacement entre littérature de création et littérature de consommation. Cela a conduit à l'invention du terme péjoratif de littérature "light», comme si expansion éditoriale impliquait dévaluation esthétique. Le rejet des écoles et la multiplication des esthétiques individuelles dans le mouvement dit de la «Nueva Narrativa Española» ont entraîné le refus de toute classification. Au niveau formel, la redéfinition des modèles romanesques se double de la multiplication des sous-genres (policier, enquête, aventure, érotisme, etc.) dont l'exploitation caractérise certains romanciers, d'Eduardo Mendoza à Soledad Puértolas, de Juan Millás à Jesús Ferrero. Si on y observe des modes - prédominance initiale du récit historique puis d'aventure, puis vogue de la science-fiction ou de l'érotisme, prédominance du récit d'enquête ou de suspense psychologique - tous coexistent avec la particularité supplémentaire que les frontières en sont mouvantes. Le plus souvent la création se réalise sur une subversion générique et une rupture des horizons d'attente.

Enfin, et nous touchons là au cœur de notre problématique, l'engouement que l'on constate pour l'Histoire nationale contemporaine, tant dans le monde éditorial que journalistique, a conduit à la nécessité d'un positionnement de l'écrivain pour ou contre son utilisation. On a assisté chez certains à l'exploitation du genre historique-fiction ou politique-fiction, ou encore chronique policière sur matière historique. Chez d'autres, à l'opposé, le refus d'une écriture de la mémoire historique a été clair, créant une des variantes de l'escapismo, définitoire de la postmodernité. Il s'agit, pour un auteur, de la tendance à « échapper » à une réalité face à laquelle on pourrait attendre un engagement de sa part. Cette non-implication existe également dans d'autres domaines que celui de l'introduction, ou non, de la matière historique ; c'est le cas de la préoccupation pour des problèmes sociaux et politiques, par exemple, qui disparait souvent derrière l'analyse d'une intériorité individuelle. Mais dans la mesure où le passé conditionne une façon d'être au présent, y échappe-t-on vraiment ou tout n'est-il que travail de reconstruction?

Pour notre réflexion sur l'Histoire dans le roman, nous avons choisi de nous intéresser à la production espagnole après 1975 pour deux raisons. D'abord parce que la mort du Général Franco constitue pour le pays une date "historique ", donc remarquable. Et ce, même si les analystes savent bien que la situation sociale et économique n'a pas attendu ce moment précis pour évoluer et que le processus historique de démocratisation qui a pu s'enclencher à ce moment n'a pas constitué une rupture brusque. Mais il a permis une actualisation progressive, marquée par des événements ponctuels. Egalement, parce que 
si l'on imagine que le système littéraire d'un pays est d'une manière ou d'une autre soumis à la remise en question globale des règles d'organisation du territoire national, comme l'a démontré Emmanuel Bouju ${ }^{7}$ dans une précieuse étude sur les modèles romanesques dans l'Espagne post-franquiste, cette date constitue aussi un point de départ d'une modification potentielle. Car la démocratisation du pays est bien plus qu'un simple fait historique qui concerne les principes et les perspectives de l'organisation politique et sociale. Certes les structures amorcent leur changement; mais ce qui entreprend une évolution fondamentale, c'est la manière dont la société espagnole se conçoit et se pense elle-même.

C'est donc à partir de cette date charnière et spécifique que nous avons voulu envisager l'intégration de l'Histoire récente dans le roman. Nous appellerons ainsi celle pour laquelle il peut subsister des témoins vivants.

En effet, l'écriture de cette Histoire revêt un aspect particulier parce que la mémoire peut être vue comme la conscience rétrospective qu'a celui qui pense maintenant d'être le même que celui qui a pensé à un autre moment du passé. Ou encore parce que la mémoire se base sur le souvenir ou « quasi-souvenir » selon l'expression de Shoemaker, c'est-à-dire se souvenir d'avoir eu telle expérience ou que quelqu'un a eu cette expérience.

En revanche, nous ne fixerons aucune limite événementielle ou temporelle pour la limite supérieure de ce qu'on appelle le passé récent. Nous entendons celui-ci comme tout moment ayant précédé le moment de l'écriture.

En questionnant le rapport du passé récent au présent, nous n'ignorons pas le fait que plus le passé est lointain, mieux c'est pour en assumer les responsabilités, et inversement, plus le passé est proche, plus il est difficile de trouver pour soi-même un positionnement. Lorsqu'on regarde en arrière, on peut adopter en effet des attitudes diamétralement opposées: l'autoculpabilisation, ou la tentation de l'innocence, avec absence totale de culpabilité. Pour l'écrivain, cela aura une importance particulière face à des événements ou situations historiques conflictuels ou controversés.

Globalement donc, après 1975, le roman opère un retour sur le passé sur lequel il se penche et réfléchit. Le processus d'effacement de l'identité, accompli sous la dictature franquiste, ne disparaît pas à la mort de Franco et ses effets persistent dans l'Espagne démocratique. Et c'est lorsque des romanciers procèdent à la mise en récit de fragments d'Histoire, lorsqu'ils évoquent des moments de ce processus, qu'ils approchent d'une voie d'écriture du présent. Et ce sera même le cas pour les plus jeunes d'entre eux qui peuvent ne pas avoir vécu cette période mais éprouver le besoin d'en faire le récit pour se l'approprier et ensuite se trouver dans leur réalité.

En 1975, on recense de multiples déclarations de romanciers comme Juan Benet, Camilo José Cela, Gonzalo Torrente Ballester, Juan Marsé, Rosa Chacel, Manuel Vázquez Montalbán apportant leur contribution au débat autour d'un certain nombre d'obsessions particulières à l'Espagne au sortir de la dictature. Pour l'écrivain, il y a prise en compte dans l'acte créateur de contraintes extra-littéraires qui ont évolué. Le lien entre écriture et censure, en particulier la nécessité de procéder à des publications à l'étranger ou encore les effets de la censure latente et de l'autocensure, n'a plus cours. Par ailleurs, l'emprise de la réalité sur le travail littéraire prend d'autres formes. Dans quelle mesure y 
a-t-il imposition de choix d'écriture par la réalité ? Comment la réalité devient-elle objet littéraire? En fait la réalité, même si elle est autre, demeure un point de repère obligé de l'écrivain.

Nous citerons à cet égard trois grands romanciers ${ }^{8}$, dont les positions antagonistes montrent qu'il y a bien questionnement autour du rôle de la littérature en matière de compréhension du monde.

Pour Camilo José Cela, l'écrivain a un rôle de régulateur et de témoin :

Je crois que le romancier est un peu le moraliste de l'époque dans laquelle il vit, et en même temps son biographe.

A l'opposé, Juan Benet refuse toute idée d'engagement :

Ce qu'il y a de mieux à faire, c'est de ne camper sur aucune position. Je suis en train de créer dans le secret une doctrine de la littérature non-engagée.

Pour Manuel Vázquez Montalbán, l'écriture est une forme de résistance:

Pour moi, écrire est intimement lié à un exercice constant de prise de conscience d'une réalité personnelle et collective. C'est une réalité qui se porte au secours de l'identité, personnelle et collective, enterrée sous une fausse identité créée par la politique.

Ainsi, alors que les écrivains cherchent à se démarquer du réalisme social des années 50 ou de sortir de la phase de préoccupation excessive pour l'expérimentation formaliste des années 60 , le débat est toujours vif quant au réalisme en littérature. Mais la question qui nous occupe est la nature du rapport à la réalité. Pour Emmanuel Bouju, qui a procédé à une étude statistique sur les romans de la période 1972-86, il y aurait un décalage entre les directions que suit le roman espagnol depuis 1975 et ce qu'elles pourraient être au regard du bouleversement historique qu'a connu l'Espagne.

Qu'en est-il en ce qui concerne le rapport passé-présent?

Lorsqu'on analyse les choix d'écriture ${ }^{9}$ qui sont faits entre 1972, période de prétransition, et 1986, entrée dans la consolidation démocratique, on constate en premier lieu une fictionnalisation de l'Histoire.

Ce choix, significatif, consiste dans l'ancrage explicite de l'objet principal du récit au sein d'une période historique déterminée. Nous entendons par là les romans qui font preuve d'un choix thématique historique - Guerre Civile, apprentissage dans l'après-guerre, avant 1975 ou moeurs de la transition et diverses situations de la modernité - mais aussi qui font état dans leur forme d'un procédé de configuration de l'Histoire dans le récit. S'agissant d'histoire récente, la guerre Civile est le repère principal de la chronologie du $\mathrm{XX}^{\mathrm{e}}$ siècle, mais bien entendu, elle est une possibilité et non un impératif.

Les romans ayant un explicite ancrage historique dans le $\mathrm{XX}^{\circ}$ siècle - avant-guerre, Guerre Civile, après-guerre - sont majoritaires durant toute la période. Cet ancrage apparaît comme plus nécessaire après 1976 car leur part passe de 55\% à 70\% du corpus entre 1972 et 1986.

Nous pouvons affiner cette idée en examinant le point d'ancrage historique. Majoritairement, il s'agit de l'après-guerre. Cette période acquiert au fil du temps une prédominance écrasante dans les romans dits ancrés historiquement Elle passe de $58 \%$, sur la période 1972-76, à $80 \%$, sur 1982-86.

Le poids de la Guerre Civile s'amenuise progressivement entre ces dates, pour ensuite connaître un regain d'intérêt. La période d'avant-guerre reste en revanche minoritaire. 
En outre, on trouve de nombreux romans dans lesquels les thématiques historiques interviennent comme critères plus ou moins déterminants au regard du propos esthétique ou idéologique de l'œuvre. Elles peuvent aussi constituer le fondement, l'objet majeur ou un objet accessoire du récit.

Enfin, l'ancrage historique peut n'être absolument pas explicite. Le roman peut avoir une visée référentielle évidente sans que soit explicitée la référence historique.

Certes, le présent diégétique s'inscrit dans la transition démocratique à $14 \%$ sur la période 1976-82 mais déjà à 47\% entre 1982 et 1986, ce qui révèle une forte nécessité de comprendre le présent. Mais une des caractéristiques les plus marquantes du corpus romanesque de la Transition c'est l'exploitation comme fondement même du récit de la thématique de l'apprentissage de l'existence dans l'Espagne de l'après-guerre, à savoir une longue période de 35 ans. Il est à noter que le lecteur reconnaît aisément cette problématique, qu'elle soit explicite bien sûr ou implicite, si elle ne constitue pas l'objet majeur du récit, ou si elle repose sur une part de non-dit qui n'entrave cependant pas la reconnaissance. Car le lecteur de ces trente dernières années a vécu cette période et par conséquent l'apprentissage sous, envers ou contre Franco. S'il ne les a pas connues luimême, il connaît forcément ceux qui les ont vécues, car c'est au moins le cas de ses propres parents.

Si l'on observe l'évolution sur la période, le fondement du récit dans cette problématique d'apprentissage devient de plus en plus explicite, ce qui traduit que dans le champ romanesque le modèle peut à présent être ouvertement revendiqué, en tant que principe d'écriture même mis en évidence par les textes.

La Guerre Civile, quant à elle, est d'abord le point sans lequel il n'y aurait évidemment pas d'après-guerre, donc le point de départ de toute cette problématique. Or nous avons signalé qu'elle ne constituait pas en tant que telle un point fondamental d'ancrage historique explicite. Mais il est clair qu'elle se pose très souvent en fondement, explicite ou implicite, du récit.

Dans le cas des romans au fondement historique explicite, il semble judicieux d'opérer à présent une distinction entre "référence historique ", cette formulation sous-entendant un événement historique identifié et reconnaissable, et "référence fictive». L'enseignement de l'étude statistique à cet égard montre que le mode historique de référentialité diminue au profit d'une référentialité fictive. Dans ce cas, l'impératif de fidélité historique sera éventuellement minoré. L'un des cas les plus significatifs est l'invention par A. Múñoz Molina de l'univers spatial de Mágina, qui fonctionne avec des références temporelles réelles. Il se produit alors un questionnement sur la notion de véracité historique à travers celle de fidélité de la mémoire.

Le mélange de niveaux temporels au sein d'un même roman revêt à notre avis un grand intérêt. Il permet que le modèle romanesque de l'apprentissage dans l'après-guerre recoupe alors un traitement thématique des moeurs de la Transition. Un roman pourra donc être clairement inscrit dans la nouvelle Espagne démocratique sans que cela n'empêche nullement un retour sur une période antérieure, en particulier la période franquiste. L'imbrication pourra être plus ou moins forte, et parfois même étroite, entre les problématiques des deux périodes. Une réflexion fondamentale sur l'héritage existentiel de la dictature franquiste sera ainsi présentée dans les romans de J.-M. Guelbenzu, J.-J. Millás, Esther Tusquets, Soledad Puértolas et bien d'autres. 

d'influence du modèle de la Guerre Civile, c'est l'évolution qualitative du modèle. Celle-ci se fait à partir d'une thématique essentiellement référentielle et de nature historique vers une problématique plus abstraite. La Guerre Civile devient alors une représentation du conflit collectif, du déchirement social, d'une nécessaire refonte d'un système de valeurs ayant atteint ses limites. Ce paradigme dépasse alors la limite chronologique de la guerre elle-même, comme les conséquences de cette guerre débordent largement la période en question. Entre la période de référence, qu'elle soit historiquement prouvée ou avec un référentiel fictif, et la période suivante, il n'y a donc pas rupture. Il y a d'une part conséquence, d'autre part possibilité de retrouver un même modèle de situation sous un angle symbolique. Les formes d'évolution historiques affichent alors une permanence dont l'élucidation fait partie de la recherche d'une identité à construire. Elles constituent à ce titre la spécificité de l'Histoire dans le roman depuis 1975.

Nous avons déjà signalé la possible présence, dans un même roman, de deux niveaux temporels marqués. Qu'une période puisse apparaitre parce qu'elle revêt une valeur paradigmatique ou questionne un système de valeurs explique que deux thématiques complémentaires, sur deux périodes, soient mises en regard et confrontées. Par exemple, Beatus ille de A. Múñoz Molina, associe des thématiques concernant la Guerre Civile en même temps que les périodes post-franquistes. Il n'est donc pas surprenant que, s'il est possible d'associer deux niveaux temporels et parfois deux problématiques liées à deux périodes historiques, à deux niveaux du passé - par exemple Guerre Civile et aprèsguerre -, le même modèle puisse fonctionner à deux niveaux temporels, l'un d'eux étant le présent. Et l'idée de "modernité » ou d'existence dans la société contemporaine trouvera à se fonder sur une confrontation des héritages et des réalisations. Il s'agit seulement de déplacer les repères historiques et de conserver le modèle analytique ou d'organisation du récit sur une double temporalité.

Selon cette modalité, l'Histoire dans le roman évite l'écueil d'un " passéisme » qui peut prendre plusieurs voies. La plus connue est de surévaluer l'essence du passé, de le considérer comme le territoire quasi privilégié du discours historique, selon l'idée que les faits se comprennent mieux plus on est loin. Mais la voie la plus grave, parce qu'elle est cachée, serait que le récit historique assure seul la revendication, à laquelle par principe on ne peut rien objecter, de la mémoire.

1982 et 1986 les 4/5èmes des romans inscrits dans l'après-guerre. Qui plus est le contexte de la Transition n'apparaît pas seulement comme un point d'ancrage référentiel, mais également comme le fondement d'une réflexion sur les mœurs qui lui sont propres. En outre, si cette thématique est exploitée de façon majeure, elle prend rarement la forme d'une référence historique.

L'organisation spécifique du roman autour d'une problématique historique, selon les variantes de la Nueva Narrativa Española qui ont été expliquées plus haut, n'est en outre pas unique. Les nouveaux modèles romanesques se fondent aussi sur d'autres éléments identifiables. On repère le centrage sur un petit nombre de personnages, surtout en ce qu'ils représentent une relation interpersonnelle. Le couple est la figure centrale chez J.-J. Millás, E. Tusquets, J.-M. Guelbenzu, etc., la famille est le pivot chez Almudena Grandes, Soledad Puértolas... Parfois la focalisation dans l'espace romanesque n'est qu'individuelle comme chez Alvaro Pombo, Lourdes Ortiz, Luis Goytisolo, etc. 

sont remplacées par un recentrage sur des segments de population, groupes à l'identité forte formant des micro-sociétés. C'est le cas pour les écrivains de la Génération X, comme Lucía Etxebarria ou José Angel Mañas, qui observent les agissements et motivations de jeunes Madrilènes de familles aisées, en rupture avec un univers parental ou social issu de l'ancien monde et dans lequel ils refusent de se reconnaître. Ces romanciers décrivent alors les palliatifs ou les solutions qui sont propres à ces microsociétés, mais qui toutes, au moins pendant un temps, s'érigent par opposition aux règles en vigueur dans l'univers dont les personnages sont issus.

Et A. Múñoz Molina revendique dans son échelle de valeurs cette non-séparation entre présent et passé, entre roman et Histoire :

Quel que soit son sujet, un livre, s'il est bon parle de son propre temps. Le présent contient tous les instants du passé ${ }^{10}$.

Donc nous pourrions considérer que le roman contenant de l'Histoire évite au moins deux des trois limitations que Paul Veyne ${ }^{11}$ juge préjudiciables au récit historique : l'opposition du contemporain et de l'historique, que vous venons de démonter et la convention du « continuum », puisque le roman associe sans nécessité de causalité et sans craindre la fragmentation différents niveaux temporels. En revanche, il ne renonce pas à l'optique factuelle, voire anecdotique.

ous avons donc précisé que la notion d'Histoire ne comportait pas en elle-même un laps de temps qui serait nécessaire pour qu'un moment passé accède au rang d'historique, ce qui rend floue la frontière du passé et donc de l'Histoire. Nous avons vu aussi que le passé pouvait surgir à partir d'un référentiel historique ou fictif. Enfin, nous avons montré que l'Histoire pouvait avoir une portée problématique et pas seulement anecdotique et qu'en cela, elle transcendait la barrière passé/présent. Ces considérations faites, nous allons à présent observer quelques modalités d'intégration de l'Histoire récente dans le roman espagnol de l'après-franquisme.

Quelques modalités

51 Si nous considérons l'introduction de l'Histoire passée dans le roman selon une gradation quantitative, le cas extrême serait que la totalité de la diégèse se déroule dans le passé et que la narration soit menée selon le point de vue d'un narrateur situé dans le passé. Mais il est aussi possible que la narration s'enracine dans le présent et que le récit concerne, dans une plus ou moins forte mesure, le passé. Le romancier dans le présent peut en effet avoir un souvenir direct ou quasi direct d'un passage plus ou moins précis de l'Histoire.

Cela étant dit, nous choisissons ici une optique qualitative. La gradation se fait alors entre la référence vague à une époque, l'introduction d'événements marqués d'une époque et enfin le travail sur ces événements. Par conséquent, notre étude des modalités d'intégration de l'Histoire dans le roman ne comporte pas une image rigide du passé, comme s'il s'agissait d'un territoire à découvrir ou à conserver. Il s'agit plutôt de les considérer comme la scène où s'élabore la construction.

Notons que les media ont eu à cet égard un rôle déterminant ces 25 dernières années. Ils sont devenus des organes de la mise en histoire ${ }^{12}$. En effet, l'histoire de la culture est devenue un réservoir à partir duquel se nourrissent les pages culturelles des journaux, et ce avec d'autant plus d'impact que nombre de romanciers d'aujourd'hui sont, ou ont été, journalistes ${ }^{13}$ - une majorité à la fin des années 70 -, ou encore sont critiques ou éditeurs. Premier niveau : l'Histoire comme décor (Melocotones helados de Espido Freire) 

la manière habituelle de nous relier à ce qui s'est produit. Trouver « de » l'Histoire dans le roman ne surprend donc plus et parfois ne se remarque même pas. Par ailleurs, la répétition régulière d'éléments du passé, dans les media ou les romans, a comme conséquence une certaine perte de l'aura de la mémoire. En outre, le développement de la technologie a permis la rénovation des supports et donc la récupération d'éléments du passé comme autant d'éléments intégrés au présent ${ }^{14}$. Ce faisant se produit une disparition de toute trace temporelle de la réalité concrète des objets, comme si l'on procédait à un nettoyage du temps sur les souvenirs, visant à annuler l'effet du temps, " ce grand sculpteur ", comme le disait Marguerite Yourcenar. révolu, c'est-à-dire abandonné et dépassé, mais une modalité, légèrement anachronique seulement, du présent.

massive du passé dans le présent modifie l'expérience de ce dernier. Le retour constant sur ce qui a été permet une assimilation lente et efficace de l'expérience, qu'elle soit la sienne propre ou celle d'un autre. On assiste donc à une expansion du présent qui s'alimente en grande partie du passé. Donc un roman sur le présent peut intégrer naturellement et sans questionnement temporel volontaire des éléments d'un passé récent. On peut alors voir ces introductions dans le roman non pas comme une mémoire artificiellement utilisée comme décor, mais au contraire comme le résultat d'un présent élargi.

C'est le cas de Melocotones helados de Espido Freire. Ce roman, qui met en scène une jeune femme en fuite pour tenter d'échapper à une menace de mort, commence par ces mots : «Il existe beaucoup de façons de tuer une personne et d'échapper sans culpabilité (...) Il existe aussi une manière ancienne et simple: l'expulsion de la personne haïe de la communauté, l'oubli de son nom ${ }^{15}$ ». Ici l'oubli est impossible; les souvenirs récurrents de pêches glacées dégustées pendant la Guerre Civile envahissent la narration, comme s'il s'agissait du seul repère fiable pour ce personnage d'exilé volontaire dans une autre ville. Mais la récupération de la mémoire familiale n'est pas facile pour autant et l'histoire individuelle de ce personnage se mêle à celle d'une cousine portant le même nom qu'elle. Le référent historique est ponctuel, fictif, anecdotique, mais il est là, servant de repère pour dire le personnage dans ce qu'il est maintenant, sans pour autant être recherché pour ce qu'il est, sans volonté de récupérer une mémoire historique.

On peut considérer que ce type de romans fait partie de ce retour au passé insidieux qui se produit de toutes parts dans la société. L'ensemble de ces éléments du passé constitue une invasion qui laisse peu de place aux individus pour se souvenir ou rechercher par eux-mêmes, comme si leur mémoire se trouvait désactivée. Certes, le passé s'en trouverait peut-être récupéré, dans le but de légitimer un avenir, mais cette récupération risquerait d'entraîner la disparition de la mémoire individuelle.

Nous touchons donc ici au problème de l'autonomie de la mémoire. Celle-ci agit à deux niveaux en ce qui concerne notre sujet de réflexion. L'un est celui du lecteur de roman historique ou contenant de l'Histoire, si l'on considère que les romans de la mémoire concourent à produire cette surabondance de références au passé dont regorge la société actuelle! Mais on peut aussi estimer que le roman historique est l'une des sources où l'individu cherche à puiser pour son travail de mémoire. 
60 L'autre niveau est celui du romancier quand il effectue, par ce biais, sa propre recherche du passé pour bâtir son présent.

Deuxième niveau : les romans de la mémoire

61 Ce problème d'autonomie de la mémoire se posera particulièrement pour le lecteur dans le cas des romans de la mémoire. Si l'on considère, comme nous l'avons montré ci-dessus, que la quête du passé constitue une base pour la construction d'une identité, on peut se demander s'il n'y pas danger. Danger à ce que l'homme-lecteur puisse fonder sa construction identitaire sur une connaissance historique dont il ignore la part de véracité et de fiction, puisque, s'agissant d'un roman, il y a forcément une part de fiction et puisque chaque auteur élabore en fait sa propre mythologie historique.

En outre, si les romans contenant de l'Histoire peuvent constituer une voie pour l'homme-romancier pour ne pas se laisser téléguider, concernant l'histoire récente et donc à portée de mémoire individuelle humaine, renoncer à se souvenir par soi-même, que ce soit par ennui ou par saturation, revient en quelque sorte à abdiquer. Abdiquer son droit à contrôler la sélection d'éléments qui doivent être conservés, et laisser à d'autres, souvent toujours les mêmes, de fixer « le " passé.

Ce décalage au niveau de l'autonomie de la mémoire se produit en chaîne: entre les sources historiques (« fiables»?) et le romancier appliqué, entre le roman « vraiment historique » et le lecteur curieux, et ainsi de suite.

Deux conséquences sont alors envisageables, l'une négative, l'autre positive.

Le risque majeur est d'atteindre un objectif souvent rêvé par les pouvoirs : homogénéiser la mémoire et imposer un récit unifié du passé. Car par exemple, les sociétés totalitaires n'ont pas imposé l'oubli, comme l'a montré Jacques Le Goff ${ }^{16}$, mais une certaine mémoire. Odo Marquard dit à ce propos que la liberté n'est pas simplement la capacité de choix politique mais surtout la conscience que le passé est multiple ${ }^{17}$.

66 A l'opposé, les romans de la mémoire peuvent apporter une pierre à la recherche historique "scientifique " en constituant un apport majeur à ce qui ne se dit pas. Par exemple, ils contribuent à retrouver et à diffuser la voix des vaincus dans le cas de la guerre civile ou encore celle de ceux qui ont fait les beaux jours du régime totalitaire qui a suivi, bien que cette possibilité soit plus délicate à mettre en place car elle apparaît comme moins bien pensante sous un régime démocratique fondé sur le consensus... En fait, les romans de la mémoire sont en mesure de s'opposer à tous ceux qui fixent ce passé de manière unidirectionnelle, tout en n'oubliant pas que les images d'un événement ne sont pas cet événement et que le retour est impossible.

Dans les romans de la mémoire, on peut déterminer deux catégories, toutes deux dignes d'intérêt : ceux qui apportent une fausse recherche historique et ceux qui constituent un apport à la connaissance d'un moment ou d'une période.

1. Le leurre en matière de recherche historique (La verdad sobre el caso Savolta, de Eduardo Mendoza)

68 Au niveau de la production littéraire, la publication en 1975 de La verdad sobre el caso Savolta de Eduardo Mendoza provoque un véritable tournant. Il s'articule autour d'un contexte historique d'une manière inédite. Cette histoire d'un scandale politico-financier dans la Barcelone des années 1910-20 fait appel à des techniques et des références romanesques jamais utilisées jusqu'alors. Des voix narratives hétérogènes alternent : des présumés documents présentés par le juge Davidson à New York pour un procès en 1927, un récit autobiographique de Javier Miranda effectué dans les mêmes circonstances, des 
séquences de récit impersonnel. Différents types de discours juxtaposés sans unification par quelque instance identifiable. Au départ, c'est le récit autobiographique qui domine. Il s'appuie par ailleurs sur une réécriture parodique de certains discours codés. De ce collage ironique se dégage progressivement la figure d'une instance implicite, qui finit par proposer au lecteur la voie de la démystification. Elle apparaît progressivement du fait du décalage par rapport à l'horizon d'attente et de l'ironie narrative. Le titre, «la vérité sur le cas Savolta ", annonce une enquête, comme s'il s'agissait d'un roman noir à la manière de Chandler; mais on ne connaîtra jamais cette vérité promise. Le jeu sur les modes discursifs, qui participe de la mystification, fait aussi l'intérêt littéraire de ce roman. La particularité de cette oeuvre correspond à la problématique de notre réflexion ici. Le récit est imprégné d'une référentialité historique car la mise au clair d'une vérité historique est l'objectif déclaré du roman, tout comme le contexte est nettement implanté dans le passé. Mais cette référentialité est en fait abandonnée dans cet échange narratif particulier. Peut-être faut-il voir au niveau de la fragmentation de l'œuvre romanesque la traduction de la sensation d'un processus contemporain de désagrégation sociale et humaine...

2. Romans de recherche historique : Galíndez de Manuel Vázquez Montalbán (1990)

S'il y a des historiens qui s'essaient au roman historique, des romanciers tentent de remplir des trous de connaissance avec des romans centrés sur un sujet précis, à travers des destins individuels.

Nous aurions pu évoquer ici le très connu Soldados de Salamina de Javier Cercas, mais nous avons choisi de traiter l'évocation du droit à la récupération de la mémoire des vaincus dans la société démocratique à travers Galíndez, de M. Vázquez Montalbán. En effet, interviennent dans ce roman des éléments que nous avons déjà évoqués tels que les notions d'identité diachronique, d'appartenance et de relativisme historique.

71 Avec une trame qui relève du roman d'espionnage, genre qui, selon Georges Tyras ${ }^{18}$ « reflète le sous-sol de l'histoire », le romancier met en place une sorte de typologie des différents rapports à la mémoire. Il réfléchit, en fait, à la problématique que ce terme recouvre dans une période de transition démocratique douce qui s'érige sur une volonté de consensus, et qui considère comme nécessaire pour le bien du pays, pour la paix civile, la mise en sommeil d'une certaine part de la mémoire espagnole ${ }^{19}$. Car la monarchie constitutionnelle et les gouvernements autonomes nés de la Transition négociée ente les différents partis politiques refusent le retour d'un passé potentiellement conflictuel qui pourrait conduire à une rupture radicale, comme l'Espagne en a déjà connue une avec le soulèvement contre la Seconde République. Le personnage de Galíndez est connu et marqué historiquement. Cet observateur du PNV auprès de l'ONU, exilé et pourfendeur du régime franquiste, enquêteur sur le régime de Trujillo à Saint-Domingue, informateur du FBI et de la CIA, fut assassiné. Mais la lumière ne fut pas faite, ni en Espagne ni aux Etats-Unis, sur cette personnalité fortement subversive à plusieurs égards. M. Vázquez Montalbán mène l'enquête pour rétablir les faits, pour que le silence sur ce personnage historique ne soit pas le "prix de la transition ${ }^{20}$ ». En outre, la forme même reflète une réflexion sur la problématique de la mémoire, qu'elle soit personnelle ou historique. Les personnages représentent chacun une position marquée. On rencontre tour à tour l'intellectuel pour qui l'engagement n'est plus d'actualité et qui trouve son confort dans la distanciation et le renoncement, le politique pragmatique, mais amnésique, qui se désintéresse de l'histoire récente de l'Espagne, tout occupé qu'il est à construire une Espagne moderne, l'universitaire volontaire qui, dans la logique d'une éthique de la 
résistance, veut récupérer la mémoire historique au prix de son propre sacrifice, et d'autres encore. L'auteur questionne le rapport à l'histoire, les différentes visions de la recherche historique mais aussi l'écriture de l'histoire qui s'inscrit dans une vision idéologique. Ainsi, de manière directe et quasi dialectique, M. Vázquez Montalbán affiche son intention : refuser l'oubli dans une éthique de la résistance, pour que présent puisse se construire autrement que selon la mentalité «pasota», désimpliquée, de la fin du millénaire en Espagne.

3. Le devoir de mémoire selon Antonio Múñoz Molina

72 Pour cet écrivain, la littérature a un devoir de mémoire et c'est en replaçant l'histoire individuelle dans le destin collectif que le personnage, le lecteur et l'écrivain retrouvent les fondements de leur identité :

la mémoire est la matière première de la fiction : la mémoire collective est l'espace dans lequel la péripétie individuelle rejoint l'histoire de tous. Il est possible qu'une des tâches du roman espagnol soit actuellement d'utiliser la mémoire comme une provocation contre l'amnésie organisée par le pouvoir. ${ }^{21}$

Identité et mémoire sont donc unies. La quête du passé se révèle être une quête identitaire à laquelle le lecteur prend une part active pour découvrir dans le passé immédiat les racines du présent et de sa propre identité.

Christine Perez ${ }^{22}$ a montré que mémoire et désir sont les fondements des quatre premiers romans d'A. Múñoz Molina, en particulier Beatus Ille, Beltenebros, El jinete polaco. Les histoires sont rattachées à un double temps référentiel : la première, en devenir, est celle d'un personnage dont le présent tout entier est tourné vers l'investigation de la seconde, appartenant à une époque apparemment révolue. Cette quête du passé naît aussi du désir et de la mémoire du narrateur chargé de relater cette double histoire car, par un effet de miroir, le parcours régressif du personnage dans le temps est redoublé par celui du narrateur plongé lui aussi dans le passé. Ces trois romans questionnent la période de la guerre civile et de l'immédiat après-guerre, époque que le romancier n'a pas connue. Il nous est donné à travers le filtre de la mémoire des narrateurs, qui reconnaissent l'oubli comme faisant partie d'eux-mêmes. En outre, l'apparition dans les romans de personnages ayant réellement existé permet à A. Múñoz Molina de se plonger dans les mythes historiques, c'est-à-dire de laisser à entendre un décalage entre les événements authentiques et la vision que nous en avons à partir des témoignages de l'Histoire. Donc au-delà de la recherche sur un point événementiel précis, s'organise une interrogation théorique : le passé est-il falsifié - volontairement ou non - par ceux qui l'ont transmis et la connaissance du passé est-elle possible?

Troisième niveau : le refus de l'Histoire ou l'« Escapismo » (Soledad Puértolas)

Chez cette romancière, qui n'est pas un cas isolé, on ne trouve aucune référence temporelle ou nominative à un moment ou à un personnage historique, si ce n'est une date, allusion effleurée à l'arrivée des socialistes au pouvoir en 1982. Ses récits sont clairement implantés dans le présent de la Transition démocratique et le refus d'un retour sur le passé est net. Pour autant, les références à un changement de morale et de système de valeurs ne sont pas retenues. Systématiquement les générations sont opposées dans le cadre de positionnements familiaux pas toujours harmonieux. Dans Días del Arenal (1992), la structure narrative cyclique autour de la vie d'un protagoniste septuagénaire permet d'envisager le bilan d'une vie s'étant déroulée sur les périodes de tous les grands événements de la vie politique espagnole depuis la Guerre Civile. La focalisation se fait sur différents personnages qui apportent chacun un pan de la vision 
morale d'une époque de contraintes: la respectabilité et le qu'en dira-t-on chez les voisins d'un même immeuble, les idées reçues sur la possibilité pour une femme d'être poète et pas seulement épouse et mère sous Franco, les interdits moraux, etc. Et lorsque le relais du protagoniste principal âgé, Antonio, est pris par une jeune femme, Susana, qui ressent le même manque d'énergie vitale, s'ouvre la spirale d'échec et d'enfermement. Mais cela se fait sans rupture brutale puisque la continuité est assurée au niveau thématique et par une identité spatiale. La temporalité est autre, les circonstances sont autres, mais une essence profonde, humaine, perdure. Peut-être peut-on y voir une métaphore du changement de régime politique qui s'est fait sans violence, mais qui s'est produit, sans doute aucun, et qui demande que l'on trouve ailleurs les voies de la construction d'une identité pas forcément en rupture avec le passé...

Dans ce cas, ce que nous appellerons le regard « historique » sur le présent est autre chose que la reconversion de l'historien en simple chroniqueur de l'actualité. Et le romancier n'a pas à se déterminer entre auteur de roman historique ou de roman d'actualité. Nous rejetterons donc la notion de «escapismo » et considérerons qu'il s'agit d'une autre modalité, certes quantitativement allégée - mais est-ce l'essentiel? - de l'intégration de l'Histoire récente dans le roman espagnol. D'autant que cette histoire récente en Espagne, autour de la période historique charnière de la fin du franquisme qui ouvre sur une fin du millénaire démocratique est tellement contrastée que la notion de passé rejoint inévitablement et visiblement celle d'Histoire événementielle.

Conclusion

Si l'on considère l'auteur de roman de la mémoire comme une des variantes de l'historien, alors on pourra se dire que le service qu'il peut rendre à sa société n'est pas forcément de se déplacer vers un passé lointain mais au contraire de demeurer dans le présent, avec des incursions plus ou moins longues dans un passé proche.

78 En matière d'Histoire dans le roman, il n'y a donc pas rupture brutale après 1975, mais modification progressive des pratiques littéraires dans un champ où domine l'impératif économique et non plus l'opposition au pouvoir politique. Mais le recours au passé pour la constitution d'une identité reste clair. Or, selon Ortega, la préoccupation pour le passé n'a d'autre origine que notre préoccupation pour l'avenir ${ }^{23}$. Il est cet égard parlant que l'Espagne ait déclaré 2006 comme année de la mémoire historique.

Le romancier a, dans ce contexte, une fonction essentielle puisqu'il affronte la tâche de tenter d'expliciter le lieu à partir duquel nous partons, malgré l'urgence que connaît un monde aussi mouvant que celui, en devenir, de l'Espagne post-franquiste. Au moment de nous interroger sur l'intégration de l'Histoire récente dans le roman, nous avons cherché à comprendre comment, en mettant en évidence la nature des questions qui sont posées au passé. Et le recours à celui-ci est apparu fondamental, tout comme, dans une certaine mesure, la nécessité de s'en défendre.

Todorov a parlé d'un « travail de sélection et de combinaison orienté nécessairement vers la recherche, non pas de la vérité, mais du bien ${ }^{24} »$. Cette sélection, effectuée par chacun dans la construction de sa mythologie personnelle, n'est pourtant pas facile à réaliser au niveau global de la société en ces temps de saturation historiographique. Et le présent ne se défend pas toujours de l'agression du passé et de cette tendance à l'invasion. Cette défense semble pourtant nécessaire pour que, au-delà des certitudes dont notre époque est remplie, l'individu et, à un niveau plus large, la société puissent décider où ils veulent que le présent les conduise. Pour que la recherche du passé ne cache pas en réalité la disparition de la notion d'avenir. 


\section{NOTES}

1. Dont ceux du colloque organisé par le CNA sur le roman historique.

2. Touton Isabelle, « Le roman historique espagnol contemporain (1975-2000) dont l'histoire se passe au Siècle d'Or (XVI-XVIIèmes siècles) à l'épreuve de trois questions théoriques ", colloque sur le roman historique, CNA, Nice, .....2005.

3. Eco Umberto, Apostille au « Nom de la rose », Paris, Grasset, Le Livre de Poche, 1985, p.87. 4. Paul Ricoeur a souligné que le lien entre la narration et l'éthique est déjà évoqué dans la Poétique d'Aristote. Pour lui « composer l'intrigue, c'est faire surgir l'intelligible de l'accidentel, l'universel du singulier » (p. 11) in Temps et récit et La mémoire, l'histoire, l'oubli. 5. Tzvetan Todorov, Los abusos de la memoria, Barcelone, Paidós.

6. Manuel Cruz, Las malas pasadas del pasado - Identidad, responsabilidad, historia, Barcelone, Anagrama, 2005. XXXIIIème Prix Anagrama d'Essai. Manuel Cruz est professeur de philosophie contemporaine à l'Université de Barcelone.

7. Bouju Emmanuel, Réinventer la littérature - Démocratisation et modèles romanesques dans l'Espagne post-franquiste, Toulouse, Presses Universitaires du Mirail, 2002. Il revendique une recherche relevant de la sociologie du champ littéraire, des théories de la réception, de la phénoménologie ricoeurienne et de la narratologie.

8. Citations extraites de Beneyto Antonio, Censura y política en los escritores españoles, Euros, Barcelona, 1975.

9. Nous utilisons ici les chiffres tirés du précieux travail effectué par Emmanuel Bouju (op. cit).

10. « un libro, trate de lo que que trate, si es bueno siempre está hablando de su propio tiempo. El presente contiene todos los instantes del pasado ». Antonio Múñoz Molina, Le .Nouveau Roman espagnol et la quête d'identité, id., p. 267 . Traduction de C.Di Benedetto.

11. Paul Veyne, cité par Manuel Cruz, op. cit., p. 149.

12. Manuel Cruz parle de « historización ». Op.cit, p.161.

13. Rosa Montero (née en 1951) passe directement du journalisme au best-seller romanesque (Crónica del desamor). Manuel Vázquez Montalbán (né en 1939), romancierjournaliste célèbre pour sa série autour du détective Pepe Carvalho. Carlos Ruiz Zafón (la sombra del viento) pour en citer un plus récemment apparu.

14. Vidéo et DVD, musique et remasterisation, photo et digitalisation de l'image.

15. «Existen muchos modos de matar a una persona y escapar sin culpa (...). Existe también una forma antigua y sencilla : la expulsión de la persona odiada de la comunidad, el olvido de su nombre ».

16. Cité par M.Cruz, p.1 70

17. Cité par M.Cruz, p. 171

18. Le désir de mémoire. Entretien avec Georges Tyras. Vénissieux, Paroles d'Aube, 1997, p. 94.

19. Pour cette partie, nous nous référons à l'analyse effectuée par David Escobar dans « la mémoire en questions dans Galíndez de Manuel Vázquez Montalbán », Les langues néolatines, Paris, mars 2003.

20. Morán Gregorio, El precio de la transición, Barcelone, Planeta, 1992. 
21. « la memoria es la materia prima de la ficción : la memoria colectiva es el espacio en que la peripecia individual se vincula a la historia de todos. Puede que una de las tareas de la novela española sea ahora mismo utilizar la memoria como una provocación contra la amnesia patrocinada por el poder ». Antonio Múñoz Molina cité par Christine Perez, Le nouveau roman espagnol et la quête d'identité : Antonio Muñoz Molina, Paris, L'Harmattan, 2001. (traduction de C.Di Benedetto).

22. Id.

23. Cité par M. Cruz, op.cit.. p.148.

24. Cité par Manuel Cruz, op.cit., p. 175.

\section{RÉSUMÉS}

Engager une réflexion sur le «roman historique» dans l'Espagne de l'après-transition démocratique conduit ici à aborder les questions, plus vastes, d'Histoire dans le roman et de roman de la mémoire. Mais les notions de passé et de présent et leurs rapports se définissent au regard de la situation particulière de l'Espagne. L'interprétation de la place qu'occupe le roman historique dans le panorama romanesque espagnol s'enrichit alors des perspectives issues de la philosophie et de la sociologie.

Au confluent de ces démarches, des exemples tirés de la littérature contemporaine et étayés par des éléments d'analyse fictionnelle et narrative figurent trois niveaux d'intégration de l'Histoire récente dans le roman espagnol. Et une identité en devenir se dessine, alors que la mémoire historique arrive clairement sur la scène littéraire en même temps qu'elle s'insinue dans les problématiques qui occupent la société espagnole de la fin du millénaire.

Adentrarse en una reflexión sobre "la novela histórica" en la España de la post- transición democrática lleva aquí a acercarse a los temas, más amplios, de Historia en la novela y de novela de la memoria. Pero las nociones de pasado y de presente se definen en el ámbito de la situación peculiar de España, y de allí el vínculo cualitativo entre las dos. La interpretación del puesto que ocupa en la narrativa española se enriquece entonces de las perspectivas que proceden de la filosofía y de la sociología.

En el confluente de estas modalidades reflexivas, unos ejemplos, sacados de la literatura contemporánea y estudiados gracias a elementos de análisis de la ficción y de la narración destacan tres niveles de integración de la Historia reciente en la novela española. De modo que una búsqueda identitaria se define, mientras la memoria histórica llega claramente al escenario literario, al mismo tiempo que se cuela dentro de las problemáticas que preocupan la sociedad española de finales de milenio.

\section{INDEX}

Mots-clés : histoire, roman espagnol contemporain, roman historique, mémoire, guerre civile, transition démocratique

Index chronologique : XXe siècle 
AUTEUR

CHRISTINE DI BENEDETTO

Maître de conférences d'espagnol à l'Université de Nice-Sophia Antipolis, CIRCPLES. 